\title{
Classification of artifacts in 3D-TEM tomography of FePt-MgO crystalline samples studied by multislice image simulation
}

\author{
Nobuo Tanaka, Akichika Ohno and Jun Yamasaki \\ Department of Crystalline Materials Science and Ecotopia Science Institute, Nagoya \\ University, Chikusa-ku, Nagoya, 464-8603, Japan
}

Size-controlled and well-arranged magnetic nano-granular materials are very important for development of future magnetic recording devices because they show new kinds of properties which have never been obtained in bulk magnets. High resolution electron microscopy has contributed so much to these kinds of research fields, but with its limitation of two-dimensional projected images of the samples. We have applied a three-dimensional (3D) electron tomography technique to FePt nanodots embedded in single crystalline magnesium oxide $(\mathrm{MgO})$ films and succeeded in obtaining clear $3 \mathrm{D}$ images of the embedded dots with less than $1 \mathrm{~nm}$ image resolution, even though they are crystalline samples[1]. In order to interpret the successful result and study various kinds of origins to make artifacts on reconstructed 3D images, we have developed an image simulation routine for reconstruction of 3D images of those dots embedded in single crystalline films by using the multi-slice image simulation method and the $3 \mathrm{D}$ back-projection method.

FePt-MgO granular films were prepared by ultra-high vacuum deposition technique[2]. First, under a base-pressure of $6.7 \times 10^{-6} \mathrm{~Pa}, \mathrm{MgO}$ was deposited in $15 \mathrm{~nm}$ thickness using an electron heating source onto a cleaved $\mathrm{NaCl}(001)$ surface kept at $573 \mathrm{~K}$. Then platinum $(\mathrm{Pt})$, iron $(\mathrm{Fe})$ and $\mathrm{MgO}(\mathrm{t}=8 \mathrm{~nm})$ were deposited successively on the $\mathrm{MgO}$ film at $673 \mathrm{~K}$. Pt and $\mathrm{Fe}$ were evaporated from another electron beam heating source and a resistance heating one, respectively. The average thicknesses of $\mathrm{Pt}$ and $\mathrm{Fe}$ were controlled to equiatomic ratio. After the deposition, sample was kept at $673 \mathrm{~K}$ for about 10 minutes, and the cooled gradually at a rate of $10-20 \mathrm{~K} / \mathrm{min}$. TEM observations were performed with a $300 \mathrm{kV}$ TEM(TECNAI, F30) with a post-column energy filter(Gatan Imaging Filter), respectively. Image recording for $3 \mathrm{D}$ reconstruction was made on the TEM with an automated tilting recording system from -70 to +70 degree with increment of 1 degree. 3D reconstruction was performed by using IMOD software and $3 \mathrm{D}$ visualization, by AMIRA software.

In the present study, a self-made simulation routine was developed for 3D image reconstruction of various structural models based on multislice image simulation software and IMOD. The calculation was made two-dimensionally corresponding to one-axial sample rotation in the present TEM. An FePt particle embedded in $\mathrm{MgO}$ single crystalline films was modeled by using a super-cell including tilt of the $\mathrm{MgO}$ crystals as illustrated in Fig. 1(a). The calculated image intensity was presented as many thin slabs corresponding to the tilt angles indicated in the right hand side in Fig. 1(b). Fig 2 shows an example of simulated 3D image in a two-dimensional space, which shows artifacts due to the missing cone effect, moiré-fringe effect and Fresnel fringe effect. In the present study, we are, for the first time, succeeded in clarifying artifacts of 3D image of crystalline samples such as the present FePt particles embedded in single 
crystalline $\mathrm{MgO}$ films. Based on the results, we have successfully reconstructed 3D images as surface rendering images of $\mathrm{Pt}$ single crystalline catalytic clusters having double structured channels, which were prepared by using MCM-48 and previously observed by ADF-STEM tomography[3].

References

[1]N. Tanaka et al., Proc. 8APEM(\#42009) and Proc. M \& M(2004) pp. 1176CD.

[2]S. Fukami et al., Mater. Trans. JIM., 45(2004) 2012.

[3]J. Yamasaki et al., Philos. Mag., 84(2004) 2819.

[4] The present authors acknowledge Prof. O. Terasaki and Prof. R. Ryoo for giving

Pt cluster samples. The present study was partly supported by grants from Japanese government as "Development of 3D electron microscope" and "Nano-hetero granular metals and their application"
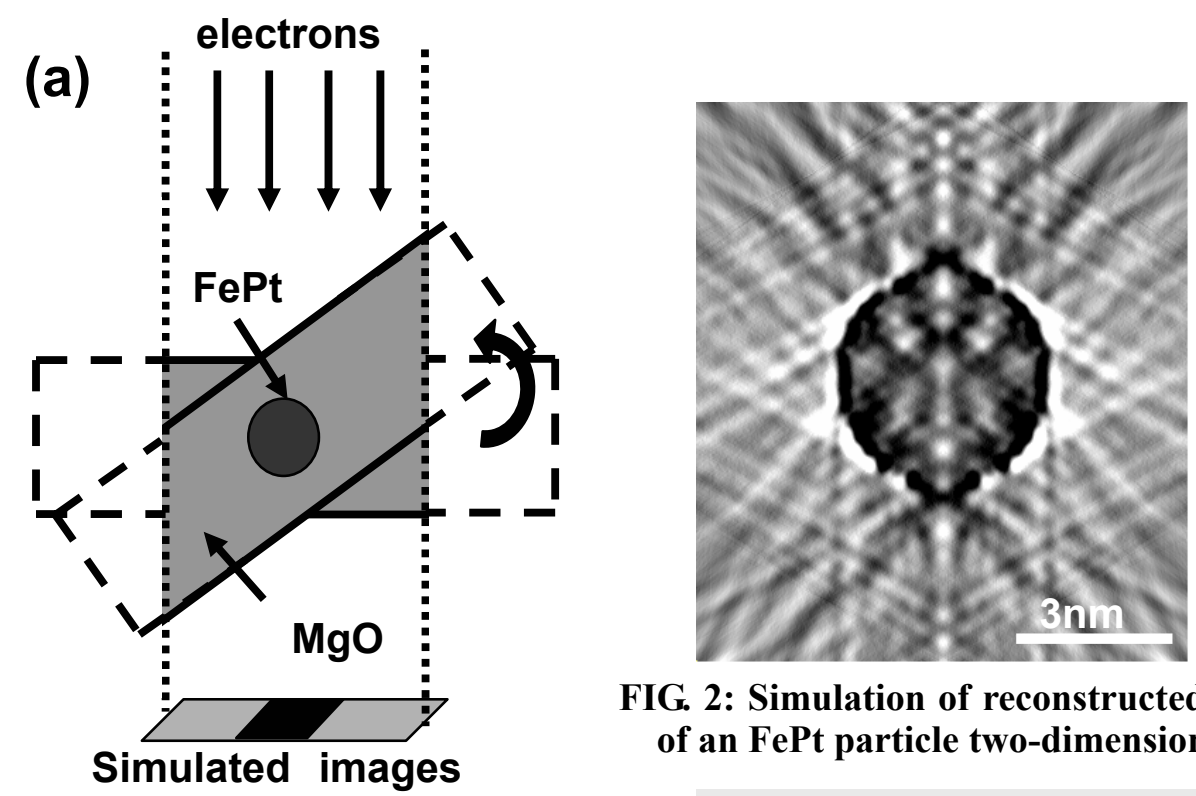

FIG. 2: Simulation of reconstructed image of an FePt particle two-dimensionally.
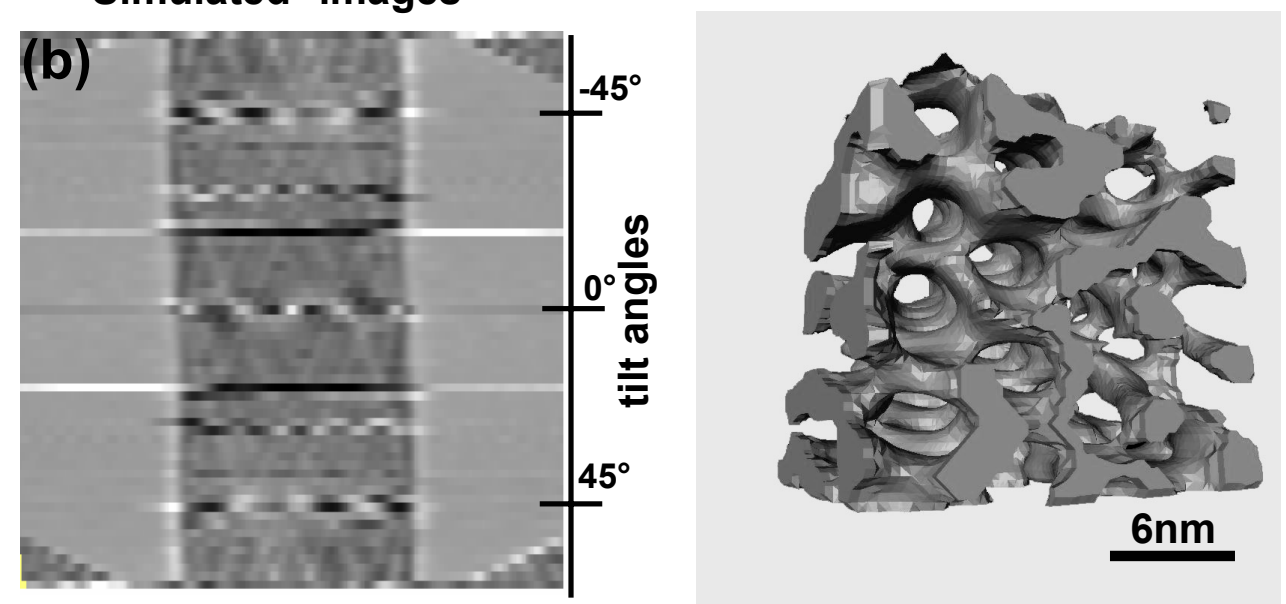

FIG. 1: (a)Illustration of a supercell for an FePt particle in $\mathrm{MgO}$ for multislice simulation, (b)Stack of slabs of calculated

FIG. 3: Surface-rendering image of a images corresponding to tilt angles. 